\title{
LOCAL MAXIMA OF THE SPHERICAL DERIVATIVE
}

\author{
By Shinji Yamashita
}

\begin{abstract}
Let a function $f$ be nonconstant and meromorphic in a domain $D$ in the plane, and let $M(f)$ be the set of points where the spherical derivative $\left|f^{\prime}\right| /\left(1+|f|^{2}\right)$ has local maxima. The components of $M(f)$ are at most countable and each component is (i) an isolated point, (ii) a noncompact simple analytic arc terminating nowhere in $D$, or, (iii) an analytic Jordan curve. Tangents to a component of type (ii) or (iii) are expressed by the argument of the Schwarzian derivative of $f$. If $\Delta$ is the Jordan domain bounded by a component of type (iii) and if $\triangle \subset D$, then the spherical area of the Riemann surface $f(\Delta)$ can be expressed by the total number of the zeros and poles of $f^{\prime}$ in $\Delta$. Solutions of a nonlinear partial differential equation will be considered in connection with the spherical derivative.
\end{abstract}

\section{Introduction.}

Let $f$ be a nonconstant meromorphic function in a domain $D$ in the complex plane $C=\{|z|<+\infty\}$. The spherical derivative of $f$ at $z \in D$ is defined by

$$
f^{\#}(z)=\left\{\begin{array}{lll}
\left|f^{\prime}(z)\right| /\left(1+|f(z)|^{2}\right) & \text { if } & f(z) \neq \infty ; \\
\left|(1 / f)^{\prime}(z)\right| & \text { if } & f(z)=\infty .
\end{array}\right.
$$

We let $M(f)$ be the set of points $z \in D$ where $f^{\#}$ has local maxima, namely, $f^{\#}(z) \geqq f^{\#}(w)$ in $\{|w-z|<\delta\} \subset D$ for $\delta>0$ depending on $f$ and $z$.

The purpose of the present paper is to investigate $M(f)$ in detail. We begin with a classification.

THEOREM 1. Let $f$ be nonconstant and meromorphic in a domain $D \subset C$ with nonempty $M(f)$. Then, the connected components of $M(f)$ are at most countable and each component is one of the following:

(I) An isolated point.

1980 Mathematıcs Subject Classification (1985 Revision). Primary 30D30. Secondary $35 \mathrm{C} 99,35 \mathrm{~J} 60$, 53A05.

Key words and phrases. Spherical derivative of a meromorphic function, Schwarzian derivative, PDE with nonlinear term.

Received August 6, 1990. 
(II) $A$ (noncompact) simple analytic are terminating nowhere in $D$.

(III) A simple closed analytic curve.

All the cases of (I), (II), and (III) actually happen; see Section 2.

Let $C_{1}(f), C_{2}(f)$, and $C_{3}(f)$ be the set of connected components of $M(f)$ of type (I), (II), and (III), respectively. Our next work is to observe them in detail.

The Schwarzian derivative of $f$ is the meromorphic function

$$
\sigma(f)=\lambda(f)^{\prime}-2^{-1} \lambda(f)^{2},
$$

where $\lambda(f)=f^{\prime \prime} / f^{\prime}$. Therefore, $f^{\#}(z) \neq 0$ if and only if $\sigma(f)(z) \neq \infty$ if and only if either $z$ is a simple pole of $f$ or $f(z) \neq \infty$ with $f^{\prime}(z) \neq 0$. The derivative $\sigma(f)$ plays an important role in

THEOREM 2. Let $f$ be nonconstant and meromorphic in a domain $D \subset \boldsymbol{C}$ with nonempty $M(f)$. Then at each $z \in M(f)$,

$$
|\sigma(f)(z)| \leqq 2 f^{\#}(z)^{2} .
$$

Furthermore, we have the following.

(IV) We can conclude $\{z\} \in C_{1}(f)$ if the inequality in (1.1) is strict.

(V) Suppose that $c \in C_{2}(f) \cup C_{3}(f)$ exists. Then, at each $z \in c$, the equality in (1.1) holds by (IV). Furthermore, the line $\left\{z+t e^{-i \theta(z) / 2} ;-\infty<t<+\infty\right\}$ is tangent to $c$ at $z$, where $\Theta(z)=\arg \sigma(f)(z)$. Moreover, there exists $\tau>0$ such that the function

$$
f^{\#}\left(z+i t e^{-i \theta(z) / 2}\right), \quad-\tau<t<\tau,
$$

has the maximum at $t=0$ and this is convex from below, that is, the second derivative with respect to $t$ is strictly negative.

The last statement in Theorem 2 is concerned with the behavior of $f^{\#}$ along the normal line of $c$ at $z$.

THEOREM 3. Let $f$ be nonconstant and meromorphic in a domain DCC. Suppose that $c \in C_{3}(f)$ exists and suppose further that the Jordan domain $\Delta$ bounded by $c$ is contained in $D$. Then

$$
(2 / \pi) \iint_{\Delta} f^{\#}(z)^{2} d x d y=Z_{\Delta}\left(f^{\prime}\right)+P_{\Delta}\left(f^{\prime}\right)-2 n,
$$

where $Z_{\Delta}\left(f^{\prime}\right)$ is the sum of all orders of all distinct zeros of $f^{\prime}$ in $\Delta$ and $P_{\Delta}\left(f^{\prime}\right)$ is that of all distinct $n$ poles of $f^{\prime}$ in $\Delta$.

The integral in the left-hand side of (1.2) is the spherical area of the Riemannian image of $\Delta$ by $f$, so that it is positive. In view of the right-hand side of (1.2) we can conclude that $f^{\#}$ must vanish at a finite number of points in $\Delta$, or equivalently, $\sigma(f)$ must have a finite number of poles in $\Delta$. If $f^{\#}$ 
never vanishes in $D$, then either $C_{3}(f)$ is empty or else each Jordan domain $\Delta$ bounded by $c \in C_{3}(f)$ is not contained in $D$.

\section{Examples and Proofs of Theorems 1 and 2.}

Before the proofs we observe that all the cases (I), (II), (III) can happen. If $f$ is a Möbius transformation $(a z+b) /(c z+d)(a d-b c \neq 0)$ considered in $\boldsymbol{C}$, then $M(f)$ is a one-point set (see the remark in Section 4). If $f(z)=z^{n}(n \geqq 2)$ is considered in $\boldsymbol{C}$, then $M(f)$ is the circle

$$
c_{n} \equiv\left\{|z|=\left(\frac{n-1}{n+1}\right)^{1 /(2 n)}\right\} .
$$

Consider the function $f(z)=z^{n}$, this time, in a domain $D$ such that both $D$ and $C \backslash D$ have the nonempty intersection with the circle $c_{n}$. Then components of $M(f)$ are of type (II) in $D$.

For the proof of Theorems 1 and 2 we shall make use of the following lemmas.

LEMMA 1. Let $g$ be holomorphic and $h$ be meromorphic in a domain $G \subset C$. Suppose that

$$
L(g, h)=\{z \in G ; \overline{g(z)}=h(z)\}
$$

has an accumulation point $a \in G$ and $g^{\prime}(a) \neq 0$. Then there exists an open disk $U(a)$ of center a such that $U(a) \cap L(g, h)$ is a simple analytic arc passing through $a$ with both terminal points on the circle $\partial U(a)$.

Proof. The case $g(z)=z$. The proof is the same as in the proof of [RW, Lemma 1]. In the general case, let $V(a)$ be an open disk with center $a$ where $g$ is univalent. Regarding $g(V(a))$ as $G, g(z)$ as $z$, and $h$ as $h \circ g^{-1}$ we can reduce this case into the case specified in the above.

LEMma 2. Let $g$ be holomorphic in a domain $G \subset C$. Suppose further that $g^{\prime}$ never vanishes in $G$. Then,

$$
M(g) \subset L(g, h),
$$

where $h=\lambda(g) /\left\{2 g^{\prime}-g \lambda(g)\right\}$. Furthermore, on each component of $L(g, h)$ the function $g^{\#}$ is constant.

Proof. Suppose that $z \in M(g)$. Taking the logarithm of $g^{\#}$ and then partially differentiating it by $w\left(\partial / \partial w=2^{-1}(\partial / \partial u-i \partial / \partial v), w=u+i v\right)$, we have

$$
\frac{\left(g^{\#}\right)_{w}(w)}{g^{\#}(w)}=\frac{1}{2} \lambda(g)(w)-\frac{\overline{g(w)} g^{\prime}(w)}{1+|g(w)|^{2}} .
$$

The value of (2.2) at $w=z$ is zero. By a simple calculation we have $h$. It 
follows from Lemma 1 that each component of $L(g, h)$ is one of the three types described in Theorem 1. Suppose that $\Lambda$ is a component of $L(g, h)$ which is not a point. Then $\Lambda$ is a simple analytic curve $w=w(t)$ in the parametric form, $a<t<b$ or $a \leqq t \leqq b$. For $w(t) \in L(g, h)$,

$$
\frac{d}{d t} g^{\#}(w(t))=2 \operatorname{Re}\left[\left(g^{\#}\right)_{w}(w(t)) w^{\prime}(t)\right]=0,
$$

whence $g^{\#}$ is constant on $\Lambda$.

For the proofs of Theorems 1 and 2 we suppose that $z \in M(f)$. Then, there is $\theta$ such that $e^{2 i \theta} \sigma(f)(z)=|\sigma(f)(z)|$; if $\sigma(f)(z)=0$, then we set $\theta=0$. There exists $\delta>0$ such that

$$
g(w)=\frac{f\left(e^{i \theta} w+z\right)-f(z)}{1+\overline{f(z)} f\left(e^{i \theta} w+z\right)}
$$

is holomorphic in $|w|<\delta$. If $f(z)=\infty$, then we set $g(w)=1 / f\left(e^{i \theta} w+z\right)$. We now have

$$
g^{\#}(w)=f^{\#}\left(e^{i \theta} w+z\right), \quad \sigma(g)(w)=e^{2 i \theta} \sigma(f)\left(e^{i \theta} w+z\right) .
$$

In particular,

$$
g^{\#}(0)=f^{\#}(z) \equiv \alpha \quad \text { and } \quad \sigma(g)(0)=|\sigma(f)(z)| .
$$

We may suppose that $g^{\prime}$ never vanishes in $|w|<\delta_{1} \leqq \delta$; actually, $\left|g^{\prime}(0)\right|=\alpha>0$. We thus have (2.2) for the present $g$, which we call (2.2P). Further differentiation of $(2.2 \mathrm{P})$ by $w$ yields

$$
\begin{aligned}
& \frac{\left(g^{\#}\right)_{w w}(w)}{g^{\#}(w)}-\left(\frac{\left(g^{\#}\right)_{w}(w)}{g^{\#}(w)}\right)^{2} \\
& \quad=\frac{1}{2} \sigma(g)(w)+\frac{1}{4} \lambda(g)(w)^{2}-\frac{\overline{g(w)} g^{\prime}(w)}{1+|g(w)|^{2}} \lambda(g)(w)+\left(\frac{\overline{g(w)} g^{\prime}(w)}{1+|g(w)|^{2}}\right)^{2} .
\end{aligned}
$$

Partial differentiation of $(2.2 \mathrm{P})$ by $\bar{w}\left(\partial / \partial \bar{w}=2^{-1}(\partial / \partial u+i \partial / \partial v)\right)$, on the other hand, yields

$$
\frac{\left(g^{\#}\right)_{w \bar{w}}(w)}{g^{\#}(w)}-\left|\frac{\left(g^{\#}\right)_{w}(w)}{g^{\#}(w)}\right|^{2}=-g^{\#}(w)^{2} .
$$

Since $0 \subseteq M(g)$, it follows that $\left(g^{\#}\right)_{w}(0)=0$, which, together with $(2.2 \mathrm{P})$ and $g(0)=0$, yields that $\lambda(g)(0)=0$. We therefore have $\left(g^{\#}\right)_{w w}(0)=2^{-1} \alpha|\sigma(f)(z)|$ and $\left(g^{\#}\right)_{w \bar{w}}(0)=-\alpha^{3}$, whence

$$
\begin{gathered}
A \equiv\left(g^{\#}\right)_{u u}(0)=-2 \alpha^{3}+\alpha|\sigma(f)(z)|, \\
\left(g^{\#}\right)_{u v}(0)=0, \\
C \equiv\left(g^{\#}\right)_{v v}(0)=-2 \alpha^{3}-\alpha|\sigma(f)(z)|<0,
\end{gathered}
$$

so that

$$
A C=\alpha^{2}\left\{4 \alpha^{4}-|\sigma(f)(z)|^{2}\right\}
$$


The Taylor expansion of $g^{\#}(w)-\alpha$ in $u$ and $v$ in $|w|<\delta_{1}$ now reads :

$$
g^{\#}(w)-\alpha=\frac{1}{2}\left(A u^{2}+C v^{2}\right)+\Gamma(u, v),
$$

where the remaining term $\Gamma(u, v)$ is a power series of $u, v$ of degree at least three. Since $g^{\#}$ has the local maximum 0 at $w=0$, and since $C<0$, it follows that $A C \geqq 0$. We therefore have (1.1).

If $A C>0$, then $g^{\#}(w)-\alpha<0$ for $0<|w|<\delta_{2} \leqq \delta_{1}$. If 0 is not an isolated point of $M(g)$, then 0 is an accumulation point of $M(g, h)$ in $G \equiv\left\{|w|<\delta_{2}\right\}$, where $h$ is as in Lemma 2. Lemmas 1 and 2 show that there is a point $w_{1} \in G \backslash\{0\}$ such that $g^{\#}\left(w_{1}\right)=\alpha$. This is a contradiction. Therefore (IV) is proved.

Suppose that $A C=0$, or $A=0$, Suppose that 0 is an accumulation point of $M(g)$. Then 0 is an accumulation point of $L(g, h)$ considered in $\left\{|w|<\delta_{1}\right\}$. Lemmas 1 and 2 then show that there exists $\delta_{3}, 0<\delta_{3} \leqq \delta_{1}$, such that

$$
\gamma \equiv M(g) \cap\left\{|w|<\delta_{3}\right\}=L(g, h) \cap\left\{|w|<\delta_{3}\right\}
$$

is a simple analytic arc ending at points on $\left\{|w|=\delta_{3}\right\}$.

Returning to $f$ we have observed that for each $z \Subset M(f)$, either (i) $z$ is an isolated point of $M(f)$ or (ii) there exists an open disk $U(z)$ of center $z$ such that $M(f) \cap U(z)$ is a simple analytic arc ending at points on $\partial U(z)$. This completes the proof of Theorem 1 .

For the proof of Theorem 2 we further analyze the case where $A C=0$ and 0 is an accumulation point of $M(g)$. Set $\gamma=\{w(t) ; a<t<b\}$. Then, $\overline{g(w(t))}=h(w(t))$, so that a short calculation shows that

$$
\begin{gathered}
\overline{w^{\prime}(t)} / w^{\prime}(t)=h^{\prime}(w(t)) / \overline{g^{\prime}(w(t))}, \\
h^{\prime} / \overline{g^{\prime}}=2^{-1} \sigma(g) / g^{\# 2} .
\end{gathered}
$$

Therefore the slope of the tangent at $w \in \gamma$ is $\tan Q(w)$, where

$$
e^{-2 i Q(w)}=2^{-1} \sigma(g)(w) / g^{\#}(w)^{2} .
$$

In particular, $Q(0)=0$. Thus, $\gamma$ has the $u$-axis as the tangent at 0 . We thus have the tangent to $c$ described in Theorem 2. Furthermore,

$$
g^{\#}(i v)-\alpha=(C / 2) v^{2}+\cdots, C<0,
$$

so that $\left(d^{2} / d v^{2}\right) F(i v)<0$ near $v=0$. This completes the proof of the theorem.

Remark. Let $z \in c \in C_{2}(f) \cup C_{3}(f)$. Rectilinear segments containing $z$ with the exception of the normal and the tangent ones to $c$ are expressed by

$$
\Lambda(\beta) \equiv\left\{\varphi_{\beta}(t) ;-\tau(\beta) \leqq t \leqq \tau(\beta)\right\},
$$

where $0<\beta<\pi / 2$, and $\varphi_{\beta}(t)=z+(1+i \tan \beta) t e^{-i \theta(z) / 2}$. It is now easy to prove 
that $f^{\#}\left(\varphi_{\beta}(t)\right)$ is convex, that is, $\left(d^{2} / d t^{2}\right) f^{\#}\left(\varphi_{\beta}(t)\right)<0$ for $|t| \leqq \tau(\beta)$, for suitable $\tau(\beta)>0$. Actually, we have in (2.5) that

$$
g^{\#}(t+i t \tan \beta)-\alpha=\frac{1}{2} C t^{2} \tan ^{2} \beta+\Gamma(t, t \tan \beta),
$$

because $A=0$. This fact shows that even in case $z \in c$, the function $f^{\#}$ attains its maximum at $z$ "in the strict sense" except along $c$.

As a further remark we let $M^{*}(f)$ be the set of points $z \in D$ where $f^{\#}$ attains the (global) maximum in $D: f^{\#}(z) \geqq f^{\#}(w)$ for all $w \in D$. Suppose that $a \in D$ is an accumulation point of $M^{*}(f)$. Then, $a \in M^{*}(f)$. Suppose that $f$ is nonconstant. Then, there exists $c \in C_{2}(f) \cup C_{3}(f)$ such that $a \in c$ because $M^{*}(f) \subset M(f)$. Since $f^{\#}$ is constant on $c$ it follows that $c \subset M^{*}(f)$. Therefore we have the analogous classification: $C_{k}^{*}(f), k=1,2,3$ of components of $M^{*}(f)$.

Suppose that isolated points of $M(f)$ has an accumulation point $a \in D$. Then $f^{\#}(a)=0$ so that $a$ is not a member of $M(f)$. For the proof we suppose that $f^{\#}(a) \neq 0$. If $f(a) \neq \infty$, then $f^{\prime}(a) \neq 0$. It then follows from Lemmas 1 and 2 that there exists an open disk $U(a)$ of center $a$ such that

where

$$
L\left(f, h_{f}\right)=\left\{z \in U(a) ; \overline{f(z)}=h_{f}(z)\right\},
$$

$$
h_{f}=\lambda(f) /\left\{2 f^{\prime}-f \lambda(f)\right\},
$$

is a simple, analytic arc on which $f^{\#}$ is constant. A contradiction comes from $M(f) \cap U(a) \subset L\left(f, h_{f}\right)$. If $f(a)=\infty$, we apply the same argument to $1 / f$ with $(1 / f)^{\prime}(a) \neq 0$ to arrive at a contradiction. Since $M *(f)$ is a closed set in $D$, it is now easy to observe that the isolated points of $M *(f)$ cluster nowhere in $D$ for nonconstant $f$.

\section{Proof of Theorem 3 .}

First of all, $f^{\#}$ never vanishes on $c=\partial \Delta$. Let $\alpha_{k}, 1 \leqq k \leqq p$, be all the simple poles of $f$ on $c$ and let $\gamma_{k}, 1 \leqq k \leqq n$, be all the distinct poles of $f$ of order $\nu_{k}$

in $\Delta$. Thus, $P_{\Delta}\left(f^{\prime}\right)=n+\sum_{k=1}^{n} \nu_{k}$. Let $A=\left\{\alpha_{1}, \cdots, \alpha_{p}, \gamma_{1}, \cdots \gamma_{n}\right\}$. Let $\varepsilon>0$ be sufficiently small, and for each $\alpha \in A$, we set

and further

$$
\delta(\alpha)=\{|z-\alpha| \leqq \varepsilon\}, \quad c(\alpha)=\{z \in \Delta ;|z-\alpha|=\varepsilon\},
$$

$$
\Delta(\varepsilon)=\Delta \backslash \bigcup_{\alpha \in A} \delta(\alpha) ;
$$

this becomes a domain bounded by a finite number of Jordan curves for sufficiently small $\varepsilon$.

Set $\Phi=\bar{f} f^{\prime} /\left(1+|f|^{2}\right)$, and $\Psi=i \Phi$. Then the Green formula 


$$
\iint_{\Delta(\varepsilon)}\left(\Psi_{x}-\Phi_{y}\right) d x d y=\int_{\partial \Delta(\varepsilon)}(\Phi d x+\Psi d y)
$$

can be rewritten as:

$$
\frac{2}{\pi} \iint_{\Delta(\varepsilon)} f^{\#}(z)^{2} d x d y=\frac{1}{\pi i} \int_{\partial \Delta(\varepsilon)} \Phi(z) d z ;
$$

the line integral is in the positive sense with respect to $\Delta(\varepsilon)$.

The Laurent expansion of $f$ about $\alpha \in A$ yields

$$
f(z)=(z-\alpha)^{-N} g(z) \quad \text { in } \delta(\alpha) \backslash\{\alpha\},
$$

where $g$ is holomorphic and nonvanishing in $\delta(\alpha) ; N=1$ if $\alpha=\alpha_{k}$, while $N=\nu_{k}$ if $\alpha=\gamma_{k}$. The differentiation yields that

$$
f^{\prime}(z)=(z-\alpha)^{-N-1} h(z), \quad h(z)=-N g(z)+(z-\alpha) g^{\prime}(z) .
$$

Since

$$
\varepsilon e^{i t} \Phi\left(\varepsilon e^{i t}+\alpha\right)=\frac{\overline{g\left(\varepsilon e^{i t}+\alpha\right)} h\left(\varepsilon e^{i t}+\alpha\right)}{\varepsilon^{2 N}+\left|g\left(\varepsilon e^{i t}+\alpha\right)\right|^{2}} \rightarrow-N \text { as } \varepsilon \rightarrow 0
$$

uniformly for real $t$, it follows that

$$
\int_{c(\alpha)} \Phi(z) d z \rightarrow\left\{\begin{array}{lll}
\pi i & \text { if } & \alpha=\alpha_{k} ; \\
2 \pi \nu_{k} i & \text { if } & \alpha=\gamma_{k},
\end{array}\right.
$$

as $\varepsilon \rightarrow 0$, where the integral is in the clockwise sense. Letting $\varepsilon \rightarrow 0$ in (3.1), we now have

$$
\begin{gathered}
\frac{2}{\pi} \iint_{\Delta} f^{\#}(z)^{2} d x d y=\frac{1}{\pi i} \int_{c} \Phi(z) d z+p+2 \sum_{k=1}^{n} \nu_{k} \\
=\frac{1}{2 \pi i} \int_{c} \lambda(f)(z) d z+p+2 \sum_{k=1}^{n} \nu_{k} .
\end{gathered}
$$

On the other hand, for $\varepsilon$ small we have

$$
\frac{1}{2 \pi i} \int_{\partial \Delta_{0}(\varepsilon)} \lambda(f)(z) d z=Z_{\Delta}\left(f^{\prime}\right)-P_{\Delta}\left(f^{\prime}\right),
$$

where

$$
\Delta_{0}(\varepsilon)=\Delta \backslash \bigcup_{k=1}^{p} \delta\left(\alpha_{k}\right) .
$$

In view of (3.2) we have in $\delta(\alpha) \backslash\{\alpha\}, \alpha=\alpha_{k}$.

$$
\lambda(f)(z)=-2 /(z-\alpha)+h^{\prime}(z) / h(z),
$$

and further, for small $\varepsilon$, the holomorphic $h$ has no zero in $\delta(\alpha)$. Therefore, letting $\varepsilon \rightarrow 0$ in the left-hand side of (3.4) we have the identity: 


$$
\frac{1}{2 \pi i} \int_{c} \lambda(f)(z) d z+p=Z_{\Delta}\left(f^{\prime}\right)-P_{\Delta}\left(f^{\prime}\right) .
$$

Combining (3.3) and (3.5) we finally have (1.2) in the theorem.

\section{A lemma.}

LEMMA 3. Let $f$ be nonconstant and meromorphic in $D$. Suppose that $z \in M(f)$ and suppose further that there exist real constants $\delta>0$ and $\theta$ such that

$$
\left|\sigma(f)\left(z+t e^{i \theta}\right)\right|>2 f^{\#}\left(z+t e^{i \theta}\right)^{2}
$$

for $0<t<\delta$. Then, $f^{\#}\left(z+t e^{i \theta}\right)$ is strictly decreasing for $0<t<\delta$.

An immediate consequence is that if $z \in M(f)$ and if $|\sigma(f)(w)|<2 f^{\#}(w)^{2}$ in $|w-z|<\delta$, then there is no point of $M(f)$ in $0<|w-z|<\delta$; the result already observed in Section 2.

Proof of Lemma 3. It suffices to consider the case $z=0$ and $\theta=0$. By a technical reason we consider $G=1 / f^{\#}$. Calculations like (2.2)-(2.4) this time, yield

$$
\frac{G_{w w}(w)}{G(w)}=-\frac{1}{2} \sigma(f)(w), \quad \frac{G_{w \bar{w}}(w)}{G(w)}=\left|\frac{G_{w}(w)}{G(w)}\right|^{2}+f^{\#}(w)^{2},
$$

whence

$$
\frac{G_{u u}(w)}{G(w)}=2\left|\frac{G_{w}(w)}{G(w)}\right|^{2}+\left(2 f^{\#}(w)^{2}-\operatorname{Re} \sigma(f)(w)\right) .
$$

Therefore,

$$
\frac{d^{2}}{d t^{2}} G(z+t)=G_{u u}(z+t)>0,
$$

together with $G_{u}(z+t) \equiv(d / d t) G(z+t) \rightarrow 0$ as $t \rightarrow+0$, shows that $G_{u}(z+t)>0$ for $0<t<\delta$. Therefore $G$ is strictly increasing on the line segment.

Remark. If $T$ is a Möbius transformation, then a computation shows that $T^{\#}(w) \rightarrow 0$ as $w \rightarrow \infty$. Furthermore, $\sigma(T) \equiv 0$, so that $|\sigma(T)(w)|<2 T^{\#}(w)^{2}$ at each point $w \in C$. We can apply Lemma 2 to consider the maximum of $T^{\#}$ of a Möbius transformation without further direct calculation. Apparently, $M(T)$ is nonempty. Suppose that there are $z_{k} \in M(T), k=1,2$, and $z_{1} \neq z_{2}$. Then a contradiction follows from Lemma 3. Therefore there is only one point $z$ such that $T^{\#}(w)<T^{\#}(z)$ for all $w \in C \backslash\{z\}$.

\section{A partial differential equation with an exponential nonlinearity.}

Let $\omega$ be a real-valued solution of the differential equation 


$$
\left(\partial^{2} / \partial z \partial \bar{z}\right) \omega+a e^{\omega}=0
$$

in a domain $D \subset C$, where $a>0$ is a constant. If $f$ is meromorphic with nonvanishing $f^{\#}$ in $D$, then

$$
\omega=\log \left(2 a^{-1}\left(f^{\#}\right)^{2}\right)
$$

is a solution. Conversely, the celebrated Liouville paper [L] shows that, if $D$ is simply connected, then each solution $\omega$ can be expressed as (5.2) for a meromorphic function $f$ with nonvanishing $f^{\#}$ in $D$. A concise proof of this is given in [W] and a detailed one is given in [B1, pp. 27-28] (see also [S]). Usually one supposes the boundary condition:

$$
\lim _{z \rightarrow \zeta} \omega(z)=0, \quad \zeta \in \partial D,
$$

to (5.1), where $\partial D$ is the boundary of $D$ in $C \cup\{\infty\}$. Since $\omega$ is superharmonic in $D$, the minimum principle shows that $\omega>0$ in $D$. For the existence of the solutions for (5.1) under (5.3), in case $D$ is bounded and simply connected, see [B1, p. 197] for example.

Let $M^{*}(\omega)$ be the set of points $z \in D$ where $\omega$ has the (global) maximum :

$$
\omega(z) \geqq \omega(w) \quad \text { for all } w \in D .
$$

We show that $M^{*}(\omega)$ is a finite set if $D$ is simply connected and (5.3) holds. First, no point-sequence extracted from $M^{*}(\omega)$ accumulates at any point of $\partial D$. Consider $M(f)$ for $f$ in (5.2). Theorem 3 shows that $C_{3}(f)$ is empty. Suppose that $c \in C_{2}(f)$ exists. If $c \cap M^{*}(\boldsymbol{\omega})$ is nonempty, then $c \subset M^{*}(\omega)$ because $f^{\#}$ is constant on $c$. This is a contradiction. Since $M^{*}(\omega) \subset M(f)$, it follows that each point of $M *(\omega)$ is an isolated point of $M *(\omega)$. The isolated points of $M^{*}(\omega)=M^{*}(f)$ cannot accumulate at any point of $D$. Therefore $M^{*}(\omega)$ is a finite set.

We return to (5.1) for general $D$. Let $M(\omega)$ be the set of points $z \in D$ where $\omega$ attains local maxima: $\omega(z) \geqq \omega(w)$ for $w$ in an open disk $U(z) \subset D$ with center $z$. Restricting (5.2) to $U(z)$ we have again a meromorphic function $f$ in $U(z)$ where $(5.2)$ is valid. By the local observation of $M(\omega)$ one can easily obtain the $\omega$-counterpart of Theorem 1. Namely, the components of $M(\omega)$ are at most countable and are classified into the three types (I), (II), and (III), described in Theorem 1. Apparently $M(\omega) \supset M^{*}(\boldsymbol{\omega})$.

As C. Bandle [B2, p. 231] (see also [B1, p. 29]) pointed out, we have

$$
\sigma(f)(z)=\frac{\partial^{2} \omega(z)}{\partial z^{2}}-\frac{1}{2}\left(\frac{\partial \omega(z)}{\partial z}\right)^{2}
$$

for (5.2) in $D$, so that, the inequality

$$
|\sigma(f)(z)| \leqq 2 f^{\#}(z)^{2} \quad \text { at } \quad z \subseteq D
$$

reads 


$$
\left|\frac{\partial^{2} \omega(z)}{\partial z^{2}}-\frac{1}{2}\left(\frac{\partial \omega(z)}{\partial z}\right)^{2}\right| \leqq a e^{\omega(z)} .
$$

\section{The Gauss curvature.}

Let $u$ be a nonconstant, real-valued, and harmonic function in a domain $D \subset C$. Then $u$ defines the surface or the graph: $\{(x, y, u(x, y)) ;(x, y) \in D\}$ in the space. The Gauss curvature $K(z)$ at the point $(x, y, u(x, y)), z=x+i y \in D$, is then $-f^{\#}(z)^{2}$, where $f=u_{x}-i u_{y}$ is holomorphic in $D$. Our results are therefore applicable to the study of the set of points in $D$ where $K$ attains local minima. See $[G, J, K, K P, T, Y]$ on the cited Gauss curvature.

\section{REFERENCES}

[B1] C. BAndle, Isoperimetric Inequalities and Applications. Pitman, Boston-LondonMelbourne, 1980.

[B2] C. BANDLE, Existence theorems, qualitative results and a priori bounds for a class of nonlinear Dirichlet problems. Arch. Rat. Mech. Anal. 58 (1975), 219238.

[G] F. Gackstatter, Die Gausssche und mittlere Krümmung der Realteilflächen in der Theorie der meromorphen Funktionen. Math. Nachr. 54 (1972), 211-227.

[J] R. Jerrard, Curvatures of surfaces associated with holomorphic functions. Colloquium Math. 21 (1970), 127-132.

[K] F. KREYSZIG, Die Realteil- und Imaginärteilfä̈chen analytischer Funktionen. Elemente der Mathematik 24 (1969), 25-31.

[KP] E. KREyszig AND A. PendL, Über die Gauss-Krümmung der Real- und Imaginärteilflächen analytischer Funktionen. Elemente der Mathematik 28 (1973), 1013.

[L] J. Liouville, Sur l'équation aux dérivées partielles $\partial^{2} \log \lambda / \partial u \partial v \pm 2 \lambda a^{2}=0$. J. de Math. Pures et Appl. 18 (1853), 71-72.

[RW] S. RUSCHEWEYh AND K.-J. WIRTHS, On extreme Bloch functions with prescribed critical points. Math. Z. 180 (1982), 91-105.

[S] T. SuzukI, Two-dimensional Emden-Fowler equation with the exponential nonlinearity. in "Nonlinear Diffusion Equations and their Equilibrium States; Wales 1989" (N.G. Lloyd, W.G. Ni, L. A. Peletier, J. Serrin, editors), Birkhäuser, Basel-Boston-Berlin, to appear. (Also, Tokyo Metropolitan University Mathematics Preprint Series 1990: No. 9, 21 pp.)

[T] G. TAlenti, A note on the Gauss curvature of harmonic and minimal surfaces. Pacific J. Math. 101 (1982), 477-492.

[W] V.H. WESTON, On the asymptotic solution of a partial differential equation with an exponential nonlinearity. SIAM J. Math. Anal. 9 (1978), 1030-1053.

[Y] S. Yamashita, Derivatives and length-preserving maps. Canad. Math. Bull. 30 (1987), 379-384.

Department of Mathematics

TOKyo METropolitan University

Minami Osawa, Hachioji, ToKyo 192-03

JAPAN. 